\title{
NESH: A JOINT SHADOWING MODEL FOR LINKS IN A MULTI-HOP NETWORK
}

\author{
Neal Patwari and Piyush Agrawal \\ University of Utah, Dept. of Electrical \& Computer Engineering \\ 50 South Central Campus Drive, Salt Lake City UT 84112, USA \\ E-mail: [npatwari, pagrawal]@ece.utah.edu
}

\begin{abstract}
Accurate analysis and simulation in multi-hop (sensor, ad hoc, and mesh) networks requires accurate representation of physical layer fading processes. Current models either ignore fading or assume independent fading on links. In reality, the shadowing losses on two geographically proximate links are correlated by the common environment through which the radio waves travel. In this paper we propose a network shadowing (NeSh) model which connects shadowing on all links in a network to a model for the physical environment in which the network operates, thus explaining shadowing correlations between links. The NeSh model is then used to analyze connectivity in a simple multi-hop network.
\end{abstract}

\section{INDEX TERMS}

Computer network performance, Radio propagation, Modeling.

\section{INTRODUCTION}

This paper provides a model for link path losses in a multi-hop network which incorporates the correlating effects of a particular environment in which the network is deployed. Because objects in the environment cause shadowing, and many links may be shadowed by each object, shadowing losses on geographically proximate links in a network are correlated.

Multi-hop network connectivity and performance is determined by path losses. In interference, path losses between an interfering transmitter and the receiver determine SINR levels. Energy loss in power controlled networks is determined by path losses. Link diversity, path diversity, and cooperative communication schemes can improve performance but are affected by multiple link path losses. In fact, all diversity schemes are limited by correlation, yet little research has modeled the correlations in path losses in multi-hop networks.

The path loss on a link has three contributions: large-scale path loss, shadowing loss, and non-shadowing loss (due primarily to small-scale or frequency selective fading). Thus the measured received power at $j$ from transmitter $i$ can be written as,

$$
P_{i, j}=\bar{P}\left(d_{i, j}\right)-X_{i, j}-Y_{i, j},
$$

where $d_{i, j}$ is the distance between nodes $i$ and $j, \bar{P}(d)$ is the largescale (ensemble mean) dB path loss at distance $d, X_{i, j}$ is the $\mathrm{dB}$ shadowing loss, and $Y_{i, j}$ is the non-shadow fading loss in $\mathrm{dB}$. We refer to the total fading loss as $Z_{i, j}=X_{i, j}+Y_{i, j}$. Large-scale path loss is [1],

$$
\bar{P}(d)=P_{T}-\Pi_{0}-10 n_{p} \log _{10} \frac{d}{\Delta_{0}},
$$

where $P_{T}$ is the dBm transmitted power, $n_{p}$ is the path loss exponent, and $\Pi_{0}$ is the loss experienced at a short reference distance $\Delta_{0}$ (typically $1 \mathrm{~m}$ ) from the transmitter antenna. Note the largescale path loss model includes average fading loss at a distance $d$ by allowing $n_{p}>2$ to be fit to the environment. This model forces (shadow and non-shadow) fading losses $X_{i, j}$ and $Y_{i, j}$ to be zero mean random variables. Note that they might more accurately be called fading loss model error, even though they are commonly referred to simply as fading loss.

We consider a network graph $(\mathcal{V}, \mathcal{E})$ with nodes (vertices) $\mathcal{V}$ and links (edges) $\mathcal{E}$. The NeSh model is motivated by the observation that shadow fading losses $\left\{X_{i, j}:(i, j) \in \mathcal{E}\right\}$ are not independent, and when links are geographically proximate, they experience significant non-zero covariance or correlation. Correlation has been measured experimentally, and Section 2 discusses the literature and explores correlation coefficients reported in one multi-hop network measurement set.

Shadowing correlation is inextricably tied to the losses caused by shadowing by objects in the environment. Thus we model link losses as a function of an underlying shadowing field $p(\mathbf{x})$. The nature of the random field $p(\mathbf{x})$ and the shadowing loss of links as function of this field is presented in Section 3. Section 4 then applies the NeSh model to the probability of connectivity in a simple example network, and compares to the best current fading model, which considers shadowing losses to be independent. We show that the current models can dramatically over-predict connectivity. Section 5 discusses future work and conclusions.

\section{LINK SHADOWING CORRELATION DATA}

Shadowing on different links in multi-hop networks has generally been assumed to be independent [2]. However, non-zero correlation coefficients on pairs of links in multi-hop networks were reported in [3]. Shadowing correlations have also been modeled for mobile links in MANETs by Wang, Tameh and Nix [4]. In mobile cellular [5] and local area networks [6], correlated shadowing has been measured and modeled, and shown to have significant effects.

We use the link fading correlation coefficients measured in [7]. This campaign measured received powers in a sensor network which was deployed in an ensemble of fifteen randomly generated environments. The campaign used sixteen nodes deployed in a four by four grid, in a $4 \times 4 \mathrm{~m}^{2}$ area. The 'environment' was changed by using a group of attenuators positioned and oriented randomly among the nodes. In the $k$ th random environment, $P_{i, j}^{(k)}$ denotes the measured received power for pair $(i, j) \in \mathcal{V}^{2}$. Total loss $Z_{i, j}^{(k)}=P_{i, j}^{(k)}-\bar{P}\left(d_{i, j}\right)$ is the total loss measured in the $k$ th random environment. Covariance is calculated between two links 


\begin{tabular}{|c|c|c|c|c|c|}
\hline \multirow[b]{2}{*}{ Geometry } & \multicolumn{2}{|c|}{ Correlation $\rho$} & \multirow[b]{2}{*}{ Geometry } & \multicolumn{2}{|c|}{ Correlation $\rho$} \\
\hline & $\begin{array}{c}\text { Meas- } \\
\text { ured }\end{array}$ & $\begin{array}{l}\text { NeSh } \\
\text { Model }\end{array}$ & & $\begin{array}{l}\text { Meas- } \\
\text { ured }\end{array}$ & $\begin{array}{l}\text { NeSh } \\
\text { Model }\end{array}$ \\
\hline$\curvearrowright$. & $0.33 \ddagger$ & 0.21 & & -0.04 & 0.05 \\
\hline$\infty \ldots$ & $0.21 \ddagger$ & 0.17 & & $0.12 \ddagger$ & 0.10 \\
\hline$<$ & $0.23 \ddagger$ & 0.24 & & $0.08^{*}$ & 0.07 \\
\hline$\sim$. & 0.05 & 0.03 & & $0.12 \ddagger$ & 0.11 \\
\hline$\approx$ & $0.17 \ddagger$ & 0.19 & & 0.03 & 0.10 \\
\hline & -0.05 & 0.00 & & $0.21 \ddagger$ & 0.13 \\
\hline & -0.01 & 0.00 & & -0.02 & 0.08 \\
\hline & $-0.10 \dagger$ & 0.00 & & $0.23 \ddagger$ & 0.16 \\
\hline & -0.03 & 0.05 & & 0.00 & 0.05 \\
\hline & $0.18 \ddagger$ & 0.21 & & 0.06 & 0.16 \\
\hline & $0.04 *$ & 0.08 & & $0.08 \dagger$ & 0.13 \\
\hline & $0.14 \ddagger$ & 0.08 & $=$ & 0.12 & 0.16 \\
\hline 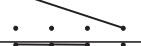 & $0.17 \ddagger$ & 0.08 & $=-$ & 0.08 & 0.00 \\
\hline.$\searrow$ & 0.05 & 0.06 & $\longrightarrow$ & 0.03 & 0.02 \\
\hline & $p=P[$ & asured & rrelation $\hat{\rho} \mid f$ & $=0]$ & \\
\hline$\ddagger$ & 0.005 & $\dagger p$ & 0.01 & $p<0.0$ & \\
\hline
\end{tabular}

Table 1. Link Geometry and Correlation Coefficients (Observed and NeSh Model)

$(i, j)$ and $(l, m)$ using the data sets $\left\{P_{i, j}^{(k)}\right\}_{k=1}^{15}$ and $\left\{P_{l, m}^{(k)}\right\}_{k=1}^{15}$.

Twenty-eight different link geometries are considered in [7]. A link geometry describes the relative coordinates of the end points of two links. For example, the link geometry in the top left of Table 1 represents a first link twice as long as, but parallel to, a second link, and with a common endpoint. According to the data, the correlation coefficient between total fading on the first and second links is 0.33 , which is statistically significant with a $p$-value of less than 0.005. Other correlation coefficients are presented in Table 1.

\section{NETWORK SHADOWING MODEL}

To model the experimentally observed characteristic of correlated link shadowing, we connect shadowing losses in one network to an underlying spatial loss field $p(\mathbf{x})$. We assume that $p(\mathbf{x})$ is isotropic wide-sense stationary Gaussian random field with zero mean and exponentially decaying spatial correlation,

$$
E\left[p\left(\mathbf{x}_{i}\right) p\left(\mathbf{x}_{j}\right)\right]=R_{p}\left(d_{i, j}\right)=\frac{\sigma_{X}^{2}}{\delta} e^{-\frac{d_{i, j}}{\delta}}
$$

where $d_{i, j}=\left\|\mathbf{x}_{j}-\mathbf{x}_{i}\right\|$ is the Euclidian distance between points $\mathbf{x}_{i}$ and $\mathbf{x}_{j}, \delta$ is a space constant and $\sigma_{X}^{2}$ is the variance of $X_{i, j}$. The contour plot of a realization of such a random field is shown in Fig. 1. We choose (3) because an exponential decay of covariance is associated with many Poisson-based processes. In the absence of other evidence, we assume that attenuation values in the environment are a derivative of a spatial Poisson process.

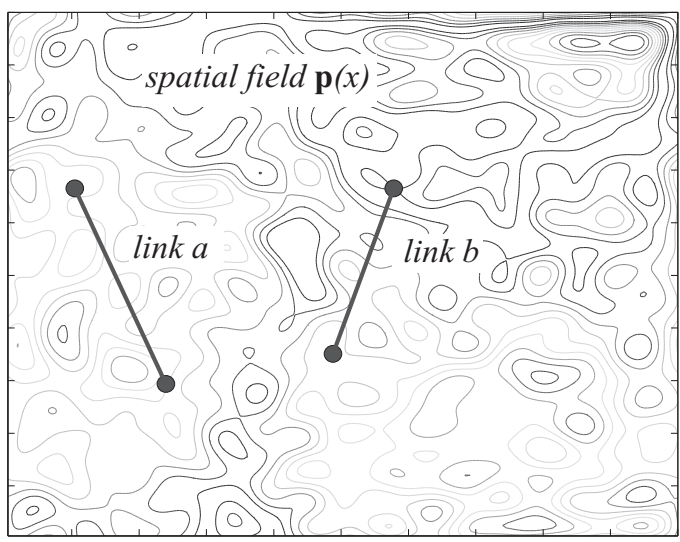

Fig. 1. A link pair in an underlying spatial loss field.

We model the shadowing on link $a=(i, j)$ as a normalized integral of $p(\mathbf{x})$ over the line between endpoints $\mathbf{x}_{i}$ and $\mathbf{x}_{j}$,

$$
X_{a} \triangleq \frac{1}{d_{i, j}^{1 / 2}} \int_{\mathbf{x}_{i}}^{\mathbf{x}_{j}} \mathbf{p}(\mathbf{x}) d \mathbf{x}
$$

Single Link Properties: The model agrees with two important empirically-observed link shadowing properties:

Prop-I The variance of shadowing (in $\mathrm{dB}$ ) on a link is approximately constant with the path length [1],[8].

Prop-II dB shadowing losses $\left\{X_{a}\right\}_{a \in \mathcal{E}}$ are Gaussian [8],[2].

The model in (4) can be seen to have Prop-II, since $X_{a}$ is a scaled integral of a Gaussian random process.

In the NeSh model, $E\left[X_{a}\right]=0$, and the variance of $X_{a}$ is

$$
\operatorname{Var}\left[X_{a}\right]=\frac{1}{d_{i, j}} \int_{\mathbf{x}_{i}}^{\mathbf{x}_{j}} \int_{\mathbf{x}_{i}}^{\mathbf{x}_{j}} R_{p}(\|\boldsymbol{\beta}-\boldsymbol{\alpha}\|) d \boldsymbol{\alpha}^{T} d \boldsymbol{\beta} .
$$

Using (3) as the model for spatial covariance, (5) is given by

$$
\operatorname{Var}\left[X_{a}\right]=\sigma_{X}^{2}\left[1-\frac{\delta}{d_{i, j}}\left(1-e^{-d_{i, j} / \delta}\right)\right] .
$$

When $d_{i, j}>>\delta$, the NeSh model has Prop-I,

$$
\operatorname{Var}\left[X_{a}\right] \approx \sigma_{X}^{2}
$$

Joint Link Properties: Since both $X_{a}$ and $X_{b}, a, b \in \mathcal{E}$, are functions of the same shadowing field $p(\mathbf{x})$, the model (4) causes non-zero covariance. The covariance of $X_{a}$ and $X_{b}$ is,

$$
\operatorname{Cov}\left(X_{a}, X_{b}\right)=\frac{\sigma_{X}^{2}}{\delta d_{i, j}^{1 / 2} d_{l, m}^{1 / 2}} \int_{\mathbf{x}_{i}}^{\mathbf{x}_{j}} \int_{\mathbf{x}_{l}}^{\mathbf{x}_{m}} e^{-\frac{\|\boldsymbol{\beta}-\boldsymbol{\alpha}\|}{\delta}} d \boldsymbol{\alpha}^{T} d \boldsymbol{\beta} .
$$

The correlation coefficient between $X_{i, j}$ and $X_{b}, \rho_{X_{a}, X_{b}}$, is

$$
\begin{aligned}
& \rho_{X_{a}, X_{b}}=\frac{\operatorname{Cov}\left(X_{a}, X_{b}\right)}{\sqrt{\operatorname{Var}\left[X_{a}\right] \operatorname{Var}\left[X_{b}\right]}} \\
& \rho_{X_{a}, X_{b}} \approx \frac{1}{\delta d_{i, j}^{1 / 2} d_{l, m}^{1 / 2}} \int_{\mathbf{x}_{i}}^{\mathbf{x}_{j}} \int_{\mathbf{x}_{l}}^{\mathbf{x}_{m}} e^{-\frac{\|\boldsymbol{\beta}-\boldsymbol{\alpha}\|}{\delta}} d \boldsymbol{\alpha}^{T} d \boldsymbol{\beta} .
\end{aligned}
$$

To generate a value for $\rho_{X_{a}, X_{b}}$, we use numerical integration. 
Non-Shadow Fading: As given in (1), received power $P_{i, j}$ has a fading component due to small-scale fading, represented as $Y_{i, j}$. We assume that $\left\{Y_{i, j}\right\}_{i, j}$ are independent. Nodes in ad hoc, sensor, and mesh networks are typically separated my many wavelengths, and small-scale fading correlation is approximately zero at such distances [9]. Further, we assume that small scale fading is independent of shadow fading.

We model small scale fading $Y_{i, j}$ with zero mean and variance $\sigma_{Y}^{2}$. While small scale fading for narrowband fading channels is typically modeled as Rayleigh, Rician, or other non-Gaussian distribution, we assume that the nodes operate using a wideband method (such as OFDM). A wideband receiver tends to average the narrowband fading at each frequency in the band. As an average of small-scale fading across a band, $Y_{i, j}$ should, by a central limit argument, appear approximately Gaussian.

Because of independence, the variance of total fading is thus

$$
\sigma_{d B}^{2} \triangleq \operatorname{Var}\left[Z_{a}\right]=\sigma_{X}^{2}+\sigma_{Y}^{2} .
$$

\subsection{Determination of Parameters}

Traditional independent link fading models have two parameters, $\sigma_{d B}^{2}$ and $n_{p}$. The NeSh model also requires these parameters, which can be obtained using the experimental method described in, or from the tables in [8]. The NeSh model introduces two additional parameters, $\sigma_{X}^{2}$ and $\delta$. In [7], these are reported to be $\sigma_{X}^{2}=0.29 \sigma_{d B}^{2}$ and $\delta=0.21 \mathrm{~m}$. The first, $\sigma_{X}^{2}$, relates the relative contribution of shadowing to total fading, and is a function of the bandwidth, center frequency, and deployment environment of the communication link. The second parameter, $\delta$, is a space constant which is proportional to the size of the objects which cause shadowing in the environment. Just like experimental campaigns have measured links in different environments to estimate $\sigma_{d B}^{2}$ and $n_{p}$ as a function of environment [8], multi-hop networks in different environments should be measured in order to estimate $\sigma_{X}^{2}$ and $\delta$.

\subsection{Discussion}

Table 1 lists the correlation coefficients computed using the NeSh model (with $\sigma_{X}^{2}=0.29 \sigma_{d B}^{2}$ and $\delta=0.21 \mathrm{~m}$ ) for each link geometry considered in [7]. In general, Table 1 shows the ability to predict positive correlations between link shadowing in many different link geometries. The model predicts both high and low correlations, and are typically close to the experimental values. We use a linear regression between the NeSh model $\rho$ and the measured $\rho$ to determine that the NeSh model predicts $80.4 \%$ of the measured variation as a function of link geometry.

As a limitation, the NeSh model does not predict negative correlation coefficients, and one link geometry does exhibit a statistically significant $\rho<0$. Measurements in [3] also measured a low magnitude negative correlation coefficient in a particular case. Our NeSh model is limited by the choice of a covariance function $R_{p}\left(d_{i, j}\right)$ in (3) which is non-negative. Future work will investigate other covariance models $p(\mathbf{x})$ which may allow negative correlation coefficients.

\section{CONNECTIVITY APPLICATION}

One of the applications of the NeSh model is in the study of the connectivity of multi-hop routes in a network. Figure 2 shows a simple three-node network whose connectivity can be readily analyzed in both i.i.d. and correlated shadowing.

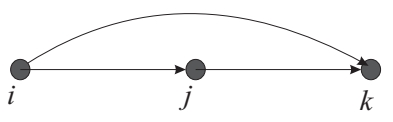

Fig. 2. Example three-node multi-hop network. Here, $d_{i, j}=d_{j, k}$.

\subsection{Analysis}

To simplify the analysis, we assume

1. Link $(i, j)$ is connected if and only if received power $P_{i, j}$ is greater than a threshold $\gamma$, and

2. No interference is present.

Assumption (1.) is a reasonable approximation in digital communication systems. For (2.), interference would accentuate the effects of the NeSh model, since path losses from an interferer will also be correlated with those between the transmitter and receiver.

We consider the connectivity of the route between nodes $i$ and $k$. Here, we use 'link' to indicate the direct communication of two nodes, and 'route' connectivity to indicate that two nodes can communicate via multi-hop through (possibly) other nodes. For route $i$ to $k$ to be connected, one of two events must occur:

- $A=$ The direct link $(i, k)$ is connected.

- $B=$ Both links $(i, j)$ and $(j, k)$ are connected.

In this terminology, event $A \cup B$ is the event of route connectivity between $i$ and $k$. Even if link $(i, k)$ is disconnected due to high shadowing, there is a chance that links $(i, j)$ and $(j, k)$ are both connected. This section shows that this 'link diversity' is not as robust as would be predicted using independent link shadowing.

We define the normalized received power as $\beta_{m, n}$,

$$
\beta_{m, n}=\frac{P_{m, n}-\gamma}{\sigma_{d B}}
$$

where $\gamma$ is the threshold received power. With this definition, events $A$ and $B$ are now given by

$$
A=\left\{\beta_{i, k}>0\right\}, \text { and } B=\left\{\beta_{i, j}>0\right\} \cap\left\{\beta_{j, k}>0\right\} .
$$

An important parameter is the expected value of $\beta_{m, n}$,

$$
\bar{\beta}_{m, n} \triangleq E\left[\beta_{m, n}\right]=\frac{\bar{P}\left(d_{m, n}\right)-\gamma}{\sigma_{d B}}
$$

where $\bar{P}(d)$ is given in (2). Intuitively, $\bar{\beta}_{m, n}$ is the number of standard deviations of link margin we have in link $(m, n)$. If we design the multi-hop network with higher $\bar{\beta}_{i, j}$ and $\bar{\beta}_{j, k}$, we will have a higher robustness to the actual fading in the environment of deployment. For example, one could set the inter-node distance to ensure that $\bar{\beta}_{i, j}=2$, and then link $(i, j)$ would only be disconnected if total fading loss was two standard deviations more than its mean. From (11) and (2), we have

$$
\bar{\beta}_{i, j}=\bar{\beta}_{j, k}, \text { and } \bar{\beta}_{i, k}=\bar{\beta}_{i, j}-\kappa,
$$

where $\kappa=\frac{10 n_{p} \log _{10} 2}{\sigma_{d B}}$.

We need to find the probability of route connectivity between $i$ and $k$, i.e., $P[A \cup B]$. We do this for both i.i.d. and NeSh models and compare the results.

Case of i.i.d. Shadowing: The probability of event $A$ is:

$$
P[A]=1-\mathrm{Q}\left(\bar{\beta}_{i, j}-\kappa\right),
$$


where $\mathrm{Q}(\cdot)$ is the complementary $\mathrm{CDF}$ of a standard Normal random variable. Under the assumption that the shadowing across links in a network is i.i.d., the probability of event $B$ is

$$
P[B]=\left(1-\mathrm{Q}\left(\bar{\beta}_{i, j}\right)\right)^{2} .
$$

The probability of route $i, k$ connectivity is thus

$$
\begin{aligned}
P[A \cup B] & =P[A]+P[B]-P[A] P[B], \\
& =1-\mathrm{Q}\left(\bar{\beta}_{i, j}-\kappa\right) \mathrm{Q}\left(\bar{\beta}_{i, j}\right)\left[2-\mathrm{Q}\left(\bar{\beta}_{i, j}\right)\right] .
\end{aligned}
$$

Case of Nesh Model: The NeSh model gives the correlation between each pair of $\beta_{i, j}, \beta_{j, k}$, and $\beta_{i, k}$. The values are

- Between $\beta_{j, k}, \beta_{i, k}$, and between $\beta_{i, j}, \beta_{i, k}: \rho_{1}=0.21$.

- Between $\beta_{i, j}, \beta_{j, k}: \rho_{0}=0.03$. Since $\rho_{0} \ll \rho_{1}$, we approximate $\rho_{0} \approx 0$ in this analysis.

Given these correlation coefficient values,

$$
P[B] \approx\left(1-\mathrm{Q}\left(\bar{\beta}_{i, j}\right)\right)^{2} .
$$

The probability of event $A$ is identical to that given in (13). The probability of route connectivity is then

$$
\begin{aligned}
P[A \cup B] & =P[A]+P[B]-P[A \cap B], \text { where, } \\
P[A \cap B] & =P\left[\left\{\beta_{i, j}>0\right\} \cap\left\{\beta_{j, k}>0\right\} \cap\left\{\beta_{i, k}>0\right\}\right] \\
& =\int_{0}^{\infty}\left[\mathrm{Q}\left(\frac{-\mu_{1}}{\sqrt{1-\rho_{1}^{2}}}\right)\right]^{2} e^{-\frac{\left(\eta-\bar{\beta}_{i, k}\right)^{2}}{2}} d \eta,
\end{aligned}
$$

and $\mu_{1}=\bar{\beta}_{i, j}+\left(\eta-\bar{\beta}_{i, j}+\kappa\right) \rho_{1}$.

\subsection{Results}

We are typically driven to minimize the probability of route failure, i.e., the probability that the route between $i$ and $k$ is not connected. Denoting this non-connectivity event as $F$, we have that

$$
P[F]=1-P[A \cup B] .
$$

We compare the $P[F]$ for both i.i.d. and NeSh models in Fig. 3. Note that the circular coverage model would result in $P[F]=0$ since links $i, j$ and $j, k$ are 'in range' for $\bar{\beta}_{i, j}>0$. The analysis shows that when a multi-hop network is designed for $\bar{\beta}_{i, j}=2$, the probability of link failure is $76 \%$ greater in the NeSh model as compared to the i.i.d. shadowing model. Increasing the reliability of the network by designing it for higher $\bar{\beta}_{i, j}$ exponentially increases the disconnect between the two models. At $\bar{\beta}_{i, j}=3$, there is a $260 \%$ difference between the two models.

\section{CONCLUSION}

We have introduced a new network shadowing (NeSh) model useful for simulation and analysis in multi-hop networks such as ad hoc, mesh, and sensor networks. The correlations between link shadowing losses are modeled as the result of the network operating in a single realization of a random underlying shadowing field $p(\mathbf{x})$. The loss on each link is proportional to a line integral of $p(\mathbf{x})$ between the transmitter and receiver locations. The model is equivalent to existing single-link shadowing models if there are only two nodes in the network, and the model extends to manylink networks which have been experimentally shown to exhibit link shadowing correlations. An example of route connectivity in a three-node multi-hop network shows that the NeSh model accounts for higher probabilities of route failure than the existing i.i.d. link shadowing model.
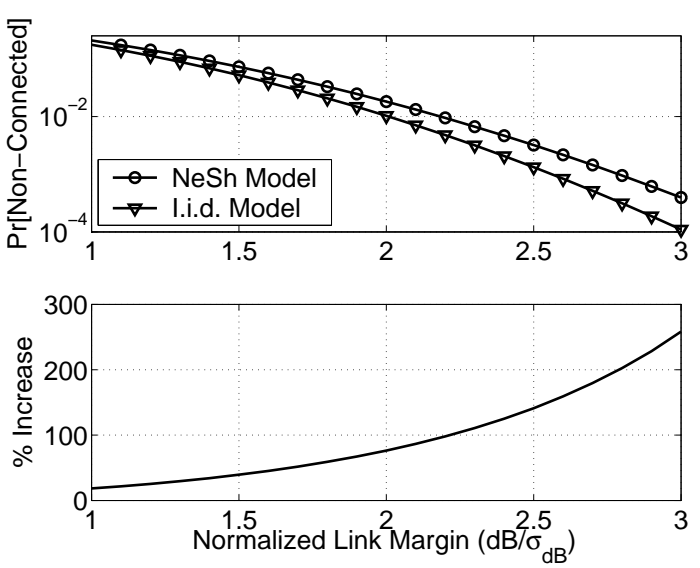

Fig. 3. Analytical $P[F]$ in NeSh and i.i.d. models, and percent increase in $P[F]$ due to correlation.

\section{REFERENCES}

[1] Homayoun Hashemi, "The indoor radio propagation channel," Proc. IEEE, vol. 81, no. 7, pp. 943-968, July 1993.

[2] Neal Patwari, Joshua Ash, Spyros Kyperountas, Robert M. Moses, Alfred O. Hero III, and Neiyer S. Correal, "Locating the nodes: Cooperative localization in wireless sensor networks," IEEE Signal Process., vol. 22, no. 4, pp. 54-69, July 2005.

[3] Neal Patwari, Yanwei Wang, and Robert J. O’Dea, “The importance of the multipoint-to-multipoint indoor radio channel in ad hoc networks," in IEEE Wireless Commun. and Networking Conf. (WCNC), March 2002, pp. 608-612.

[4] Zhenyu Wang, Eustace K. Tameh, and Andrew Nix, "Simulating correlated shadowing in mobile multihop relay/ad-hoc networks," Tech. Rep. IEEE C802.16j-06/060, IEEE 802.16 Broadband Wireless Access Working Group, July 2006.

[5] M. Gudmundson, "Correlation model for shadow fading in mobile radio systems," IEE Electronics Letters, vol. 27, no. 23, pp. 2145 - 2146, 7 Nov. 1991.

[6] Keith S. Butterworth, Kevin W. Sowerby, and Allan G. Williamson, "Base station placement for in-building mobile communication systems to yield high capacity and efficiency," IEEE Trans. Commun., vol. 48, no. 4, pp. 658-669, April 2000.

[7] Piyush Agrawal and Neal Patwari, "Correlated link shadow fading in multi-hop wireless networks," IEEE Trans. Wireless Commun., Submitted Nov. 2007, [Online] http: //span. ece.utah.edu/pmwiki/pmwiki. php?n=Main. Archives.

[8] Theodore S. Rappaport, Wireless Communications: Principles and Practice, Prentice-Hall Inc., New Jersey, 1996.

[9] Gregory D. Durgin, Space-Time Wireless Channels, Prentice Hall PTR, 2002. 